\title{
Transmisión de COVID-19 en el personal de salud del hospital Víctor Lazarte Echegaray de Trujillo
}

Marco Antonio Alfaro Angulo* 1,a

\section{RESUMEN}

Objetivo: Describir las características de la transmisión de COVID-19 en el personal de salud del hospital Víctor Lazarte Echegaray de Trujillo.

Materiales y métodos: Estudio de tipo descriptivo. Los datos fueron recolectados mediante entrevistas, fichas clínicoepidemiológicas e historias clínicas. Se identificó al personal que atendió a los pacientes con COVID-19 y a quienes adquirieron la infección y desarrollaron síntomas. Los procedimientos realizados en los pacientes fueron registrados en una lista. Los casos se describen según tipo de personal de salud y semana epidemiológica. Las frecuencias absolutas y relativas, así como la tasa de ataque, fueron determinadas. El Comité de Investigación y Ética del hospital aprobó el estudio.

Resultados: Seis pacientes hospitalizados tuvieron el diagnóstico confirmado de COVID-19. Todos ellos fueron atendidos por 45 trabajadores de la salud en procedimientos como hemodiálisis, ventilación mecánica, intubación orotraqueal, nebulización y endoscopía alta sin los equipos de protección personal. A consecuencia de ello, 38 individuos resultaron infectados y presentaron un cuadro clínico caracterizado por malestar general, tos, fiebre y dolor de garganta. Los médicos y las enfermeras fueron los trabajadores de salud más afectados.

Conclusiones: La transmisión intrahospitalaria de COVID-19 en el personal de salud fue evidente. Los trabajadores más afectados fueron los médicos y las enfermeras. La tasa de ataque fue de $84,44 \%$.

Palabras clave: Transmisión; COVID-19; Personal de salud (Fuente: DeCS BIREME).

\section{Transmission of COVID-19 among the health personnel of the Hospital Víctor Lazarte Echegaray in Trujillo}

\section{ABSTRACT}

Objective: To describe the characteristics of COVID-19 transmission among the health personnel.

Materials and methods: A descriptive research was conducted. Data were collected through interviews, clinicalepidemiological records and medical records. The personnel who treated COVID-19 patients, and those who acquired the infection and developed symptoms were identified. The medical procedures undergone by the patients were listed. The cases were described according to the type of health personnel and epidemiological week. The absolute and relative frequencies, as well as the attack rate, were determined. The research was approved by the hospital's research and ethics committee.

Results: The diagnosis of COVID-19 was confirmed in six hospitalized patients. All of them were treated by 45 health workers in procedures such as hemodialysis, mechanical ventilation, orotracheal intubation, nebulization and upper endoscopy with no personal protective equipment. As a result, 38 individuals were infected and developed symptoms, including malaise, cough, fever and sore throat. The most affected health workers were doctors and nurses.

Conclusions: In-hospital transmission of COVID-19 was evident in the health personnel, affecting most frequently doctors and nurses. The attack rate accounted for $84.44 \%$.

Keywords: Transmission; Coronavirus infections; Health personnel (Source: MeSH NLM).

1 Red Asistencial La Libertad- EsSalud, Oficina de Inteligencia Sanitaria. Trujillo,Perú.

a Médico Cirujano, Especialista en Enfermedades Infecciosas y Tropicales Maestro en Salud Pública, con mención en Epidemiología.

* Autor corresponsal. 


\section{INTRODUCCIÓN}

Los coronavirus causan enfermedades respiratorias, desde el resfrío común hasta enfermedades graves, como el síndrome respiratorio de Oriente Medio y el síndrome respiratorio agudo severo ${ }^{(1,2)}$.

El coronavirus de tipo 2, causante del síndrome respiratorio agudo severo (SARS-CoV-2), fue identificado como el agente que provocó el brote detectado en Wuhan (China) ${ }^{(3-8)}$ en diciembre del 2019. Desde entonces se ha propagado hasta convertirse en una pandemia mundial con 78145043 casos reportados hasta el 3 de enero del presente año ${ }^{(9)}$.

En el Perú, el primer caso importado de COVID-19 se presentó el 5 de marzo del 2020. Después, la enfermedad se ha propagado hasta alcanzar 1018099 casos a nivel nacional al 3 de enero. En el departamento de La Libertad se han registrado 37489 casos para la misma fecha ${ }^{\left({ }^{10}\right)}$. En la Red Asistencial La Libertad, la Oficina de Inteligencia Sanitaria ha reportado 15955 infectados, de los cuales 178 corresponden a personal de salud ${ }^{(11)}$.

El período de incubación de la enfermedad es, aproximadamente, de 1 a 14 días (en promedio, 5 días) ${ }^{(12-14)}$. El contagio se produce si una persona sana se expone al virus cuando una persona enferma tose o estornuda, por las manos contaminadas y objetos inanimados que albergan partículas virales ${ }^{(15,16)}$.

La mayoría de las infecciones son autolimitadas. El virus tiende a causar enfermedades más graves en personas mayores de 60 años o en pacientes con problemas médicos subyacentes. La enfermedad es leve en el $81 \%$ de los pacientes; grave, en $14 \%$ y crítica en el $5 \%$; y tiene una tasa de letalidad del 2,3 al $5 \%{ }^{(17)}$.

Al inicio, las manifestaciones clínicas son fiebre, tos seca, fatiga, mialgias y disnea. La neumonía parece ser la manifestación más común y grave de la infección. La dificultad para respirar se desarrolla, en promedio, a los cinco días de la infección inicial. Otros síntomas son dolor de cabeza, dolor de garganta, rinorrea y síntomas gastrointestinales ${ }^{(18)}$. El diagnóstico se confirma con la prueba de reacción en cadena de polimerasa de transcripción inversa en tiempo real (RT-PCR) ${ }^{(19)}$.

La alta transmisión de la enfermedad en el departamento de La Libertad ha propiciado que el personal de salud contraiga la infección durante la atención de los pacientes, con la probabilidad de propagar la enfermedad a otros pacientes hospitalizados y compañeros de trabajo, lo que puede ocasionar absentismo laboral y disminuir la oferta de servicios.

El artículo describe la transmisión intrahospitalaria de la infección por SARS-CoV-2 en el personal de salud del hospital Victor Lazarte Echegaray de Trujillo.

\section{MATERIALES Y MÉTODOS}

\section{Diseño y población}

Estudio descriptivo realizado en el Hospital Víctor Lazarte Echegaray, ubicado en la ciudad de Trujillo (Perú), con 1080 trabajadores considerados como personal de salud.

Los datos se recolectaron mediante entrevistas al personal de salud, revisión de las fichas clínico-epidemiológicas y de las historias clínicas, con reserva de la confidencialidad y el anonimato. Las fichas no requirieron consentimiento informado, al ser documentos epidemiológicos protocolizados. Toda persona con una prueba molecular de RT-PCR en muestra respiratoria $\mathrm{y} / \mathrm{o}$ una prueba rápida de detección de IgM/lgG positiva para COVID-19 fue considerada como un caso confirmado ${ }^{(20)}$.

Se elaboró una relación del personal que atendió a los pacientes y de quienes adquirieron la infección. Para determinar cuál fue el personal que se contagió después de atender a los pacientes, fue necesario considerar que este grupo no haya tenido contacto con otras personas enfermas de COVID-19 en el ámbito extrahospitalario y que no usaran el equipo de protección personal durante la atención. Los procedimientos realizados en los pacientes se registraron en una lista, los síntomas fueron identificados y se calculó la tasa de ataque.

\section{Variables de estudio}

Las variables epidemiológicas que se consideraron para este estudio fueron persona, lugar y tiempo.

\section{Análisis estadístico}

Se utilizaron frecuencias absolutas y relativas, y se calculó la tasa de ataque.

\section{Consideraciones éticas}

El estudio fue aprobado por el Comité de Investigación y de Ética del Hospital Víctor Lazarte Echegaray.

\section{RESULTADOS}

Seis pacientes ingresaron al área de emergencia del hospital, entre el 20 y el 31 de marzo del 2020. Al inicio, no fueron sospechosos de padecer de COVID-19, pues se desconocía si eran contactos de personas con esta enfermedad.

Durante la hospitalización, los pacientes fueron sometidos a procedimientos de hemodiálisis, ventilación mecánica, intubación orotraqueal, nebulización y endoscopía alta, en los que intervinieron médicos, enfermeras, técnicos de enfermería y tecnólogos médicos. Los casos se confirmaron con la prueba de RT-PCR para el SARS-CoV-2. Cuarenta y 
cinco individuos del personal de salud fueron identificados como contactos y, de ellos, treinta y ocho se infectaron. Los síntomas de los trabajadores infectados fueron, principalmente, malestar general, tos, fiebre y dolor de garganta. La tasa de ataque se calculó en $84,44 \%$. Los casos se presentaron desde la semana epidemiológica 13 a la 17, tal como se muestra en la figura 1.

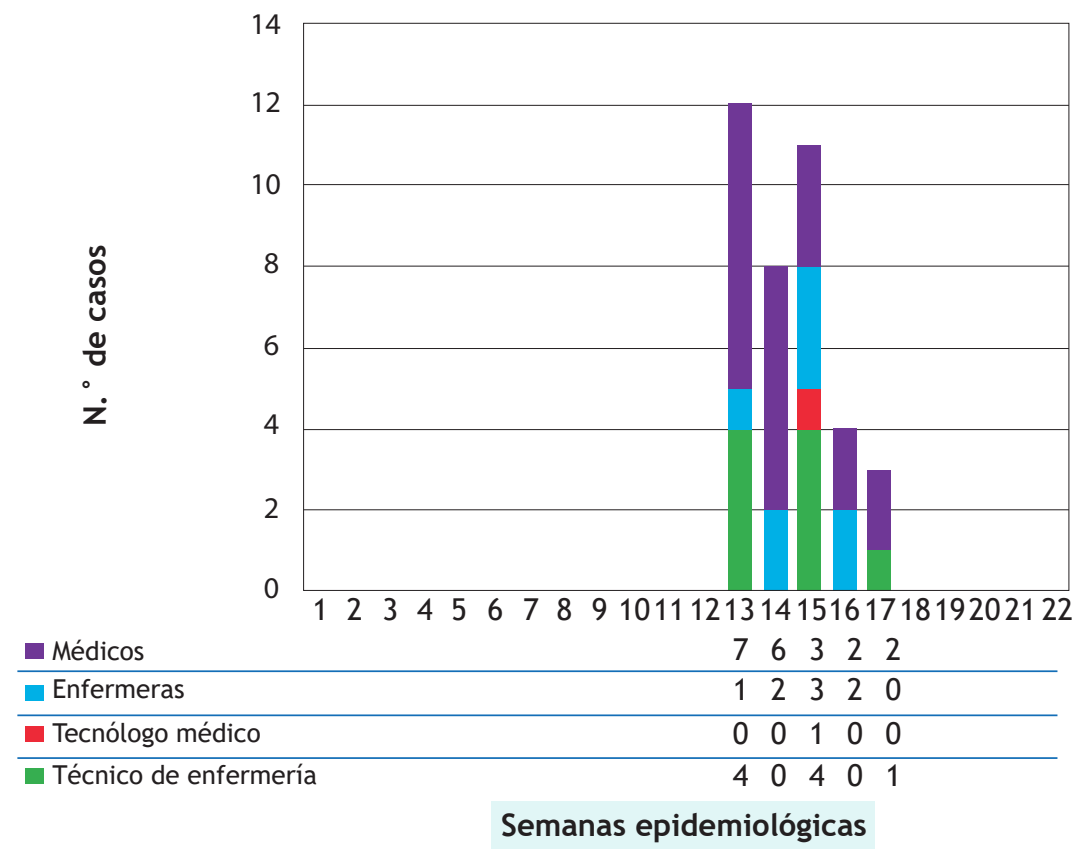

Figura 1. Casos de COVID-19 en el personal de salud por semana epidemiológica, Hospital Víctor Lazarte Echegaray de Trujillo, 2020

La mayoría de los trabajadores que adquirieron la enfermedad de COVID-19 después de brindar atención a los pacientes fueron médicos y enfermeras, con predominio de las mujeres (Tabla 1).

Tabla 1. Distribución del personal de salud que adquirió COVID-19 en el Hospital Víctor Lazarte Echegaray de Trujillo, 2020

\begin{tabular}{|lcccc|}
\hline Personal de salud & N. ${ }^{\circ}$ & Hombres & Mujeres & $\%$ \\
\hline Médicos & 16 & 12 & 4 & 42,11 \\
Enfermeras & 14 & 0 & 14 & 36,84 \\
Técnicos de enfermería & 7 & 0 & 7 & 18,42 \\
Tecnólogos médicos & 1 & 1 & 0 & 2,63 \\
Total & 38 & 13 & 25 & 100,00 \\
\hline
\end{tabular}

\section{DISCUSIÓN}

Esta investigación sobre la transmisión de COVID-19 en el personal de salud es una de las primeras publicaciones en el departamento de La Libertad y en el país; actualmente poco se sabe de la transmisión en los servicios de salud. La epidemia de COVID-19 ha afectado a la población en general y, dentro de esta, es posible el compromiso de diversos grupos ocupacionales tales como el personal de salud, que tiene un mayor riesgo de adquirir infecciones durante los brotes. Esto es más probable, sobre todo, cuando se atienden pacientes con enfermedades emergentes, con un mecanismo de transmisión desconocido o que o se está 
conociendo, como sucede en esta epidemia.

La transmisión en el personal de salud del Hospital Víctor Lazarte Echegaray ocurrió entre las semanas epidemiológicas 13 y 17, después de que atendieron a pacientes cuyo diagnóstico de COVID-19 no fue advertido, ya que en ese momento, en La Libertad, recién se estaban notificando los primeros casos.

Puesto que la enfermedad fue adquirida en el lugar de trabajo, se identificaron los procedimientos que el personal realizó a los pacientes y que facilitaron la transmisión, tales como instalación de catéter para realizar hemodiálisis, ventilación mecánica, intubación orotraqueal, endoscopía alta y nebulización. Durante estos procedimientos la exposición a los pacientes es mayor y, además, se generan aerosoles $\mathrm{y}$, por lo tanto, aumenta el riesgo de contagio. Sin embargo, hasta este momento, se conoce poco sobre los factores de riesgo para la transmisión del SARS-CoV-2 en los servicios de salud ${ }^{(21)}$.

La tasa de ataque mide la extensión de una enfermedad en grupos particulares observados durante períodos limitados; tal como se informa en este estudio ${ }^{(22)}$. En esta investigación la tasa de ataque fue $84,44 \%$; otros describen tasas de ataque menores (36\%) ${ }^{(23)}$ e incluso una nula tasa de ataque (24-27), atribuyéndose estas variaciones al uso o no de los equipos de protección personal y a su uso adecuado (28), refiriéndose a que las tasas de transmisión tienen relación con la duración de la exposición y con realizar procedimiento de riesgo, además de la disponibilidad de equipos de protección personal $(21,23,25,27,28)$.

Los médicos y las enfermeras fueron los profesionales de la salud más afectados, ya que brindaron la atención directa a los pacientes durante los procedimientos. Ellos informaron que no utilizaron los equipos de protección personal, hecho muy similar a lo que se reporta en otro estudio ${ }^{(26)}$, por lo que esta es la causa más probable de la transmisión de la enfermedad. Asimismo, los síntomas que reportamos en nuestros grupos de investigación son semejantes a otros estudios ${ }^{(28)}$.

Este trabajo de investigación describe la transmisión intrahospitalaria de COVID-19 en el personal de salud, con una tasa de ataque de $84,44 \%$ que afecta, principalmente, a médicos y enfermeras, con predominio del sexo femenino.

Finalmente, en relación a la atención de pacientes con COVID-19, las referencias consultadas para este trabajo recomiendan determinar el nivel de riesgo de exposición y enfatizan la necesidad de utilizar equipos de protección de buena calidad, con un abastecimiento permanente, uso adecuado y supervisión de su correcto empleo. Así mismo, se recomienda la detección temprana de casos, control de la fuente y su aislamiento oportuno.
Los procedimientos invasivos deben limitarse solo a los necesarios. Es necesario implementar la vigilancia epidemiológica de la transmisión en el personal de salud. De manera general, las precauciones estándar deben aplicarse y la organización de los servicios debe mejorar ${ }^{(21,26-30)}$.

Fuentes de financiamiento: Este artículo ha sido financiado por el autor.

Conflicto de interés: El autor declara no tener conflicto de intereses.

\section{REFERENCIAS BIBLIOGRÁFICAS}

1. Centers for Disease Control and Prevention. National Center for Immunization and Respiratory Diseases (NCIRD) [Internet]. 2020. Disponible en: https://www.cdc.gov/ncird/index.html

2. De Wit E, Van Doremalen N, Falzarano D, Munster VJ. SARS and MERS: recent insights into emerging coronaviruses. Nat Rev Microbiol. 2016; 14(8): 523-34.

3. Ribao H. Wuhan Municipal Health Commission's briefing on the pneumonia epidemic situation [Internet]. China; 2019. Disponible en: https://crofsblogs.typepad.com/h5n1/2019/12/wuhanmunicipal-health-commission-announces-pneumonia-epidemic.html

4. XinhuaNet.com. New-type coronavirus causes pneumonia in Wuhan: expert [Internet]. China; 2020. Disponible en: http://www. xinhuanet.com/english/2020-01/09/c_138690570.htm

5. Wang C, Hornby PW, Hayden FG, Gao GF. A novel coronavirus outbreak of global health concern. Lancet. 2020; 395(10223): 470-3.

6. Hui DS, Azhar El, Madani TA, Ntoumi F, Kock R, Dar O, et al. The continuing 2019-nCoV epidemic threat of novel coronaviruses to global health - the latest 2019 novel coronavirus outbreak in Wuhan, China. Int J Infect Dis. 2020; 91: 264-6.

7. Zhu N, Zhang D, Wang W, Li X, Yang B, Song J, et al. A novel coronavirus from patients with pneumonia in China, 2019. N Engl J Med. 2020; 382(8): 727-33.

8. Chan JF-W, Kok K-H, Zhu Z, Chu H, To KK-W, Yuan S, et al. Genomic characterization of the 2019 novel human-pathogenic coronavirus isolated from a patient with atypical pneumonia after visiting Wuhan. Emerg Microbes Infect. 2020; 9(1): 221-36.

9. Johns Hopkins - University of Medicine. Coronavirus Resource Center [Internet]. USA; 2020. Disponible en: https://coronavirus.jhu.edu/

10. Ministerio de Salud. Sala Situacional COVID-19 Perú [Internet]. Perú; 2020. Disponible en: https://covid19.minsa.gob.pe/sala_ situacional.asp

11. Red Asistencial La Libertad-ESSALUD. Sala de Situación COVID-19 de la Oficina de Inteligencia Sanitaria, Trujillo-Perú [Internet]. Perú; 2020.

12. Li Q, Guan X, Wu P, Wang X, Zhou L, Tong Y, et al. Early transmission dynamics in Wuhan, China, of Novel Coronavirus-Infected Pneumonia. N Engl J Med 2020; 382(13): 1199-207.

13. Guan W-J, Ni Z-Y, Hu Y, Liang W-H, Ou C-Q, He J-X, et al. Clinical Characteristics of Coronavirus Disease 2019 in China. N Engl J Med. 2020; 382: 1708-20.

14. Chan JF-W, Yuan S, Kok K-H, To KK-W, Chu H, Yang J, et al. A familial cluster of pneumonia associated with the 2019 novel coronavirus indicating person-to person transmission: a study of a family cluster. Lancet. 2020; 395(10223): 514-23.

15. Otter JA, Donskey C, Yezli S, Douthwaite S, Goldenberg SD, Weber DJ. Transmission of SARS and MERS coronaviruses and influenza virus in 
healthcare settings: the possible role of dry surface contamination. J Hosp Infect. 2016; 92(3): 235-50.

16. Dowell SF, Simmerman JM, Erdman DD, Wu J-SJ, Chaovavanich A, Javadi $M$, et al. Severe acute respiratory syndrome coronavirus on hospital surfaces. Clin Infect Dis. 2004; 39(5): 652-7.

17. Wu Z, McGoogan JM. Characteristics of and important lessons from the coronavirus disease 2019 (COVID-19) outbreak in China: Summary of a report of 72314 cases from the Chinese Center for Disease Control and Prevention. JAMA. 2020; 323(13): 1239-42.

18. Guan W, Ni Z, Hu Y, Liang W, Ou C, He J, et al. Clinical Characteristics of Coronavirus Disease 2019 in China. N Engl J Med. 2020; 382: 1708-20.

19. European Centre for Disease Prevention and Control. Case definition for coronavirus disease 2019 (COVID-19), as of 29 May 2020 [Internet]. Disponible en: https://www.ecdc.europa.eu/en/ covid-19/surveillance/case-definition

20. Ministerio de Salud. Alerta Epidemiológica Código: AE-16-2020 Alerta Epidemiológica ante la transmisión de COVID-19 en el Perú [Internet]. Perú; 2020. Disponible en: https://www.dge.gob.pe/ portal/docs/alertas/2020/AE016.pdf

21. Heinzerling A, Stuckey M, Scheuer T, Xu K, Perkins K, Resseger H, et al. Transmission of COVID-19 to health care personnel during exposures to a hospitalized patient - Solano County, California, February 2020. MMWR Morb Mortal Wkly Rep . 2020; 69(15): 472-6.

22. Last J. Diccionario de Epidemiología. Salvat Editores S. A.; 1989. 200 p.

23. Ghinai I, MCPherson TD, Hunter JC, Kirking HL, Christiansen D, Josh $\mathrm{K}$, et al. First known person-to-person transmission of severe acute respiratory syndrome coronavirus 2 (SARS-CoV-2) in the USA. Lancet. 2020; 395(10230): 1137-44.

24. Ng K, Poon BH, Kiat Puar TH, Shan Quah JL, Loh JW, Wong YJ, et al. COVID-19 and the risk to health care workers: a case report. Ann Intern Med. 2020; 172(11): 766-7.

25. Cheng VCC, Wong S-C, Chen JHK, Yip CCY, Chuang VWM, Tsang OTY, et al. Escalating infection control response to the rapidly evolving epidemiology of the coronavirus disease 2019 (COVID-19) due to SARS-CoV-2 in Hong Kong. Infect Control Hosp Epidemiol. 2020; 41(5): 493-8.

26. Tran K, Cimon K, Severn M, Pessoa-Silva CL, Conly J. Aerosol generating procedures and risk of transmission of acute respiratory infections to healthcare workers: a systematic review. PLoS One. 2012; 7(4): e35797.

27. Van Doremalen N, Bushmaker T, Morris DH, Holbrook MG, Gamble A, Williamson BN, et al. Aerosol and surface stability of SARS-CoV-2 as compared with SARS-CoV-1. N Engl J Med. 2020; 382(16): 1564-7.

28. CDC COVID-19 Response Team. Characteristics of health care personnel with COVID-19-United States, February 12-April 9, 2020. MMWR Morb Mortal Wkly Rep. 2020; 69(15): 477-81.

29. Center for Disease Control and Prevention. Guía provisional de EE. UU. para la evaluación de riesgos y las restricciones laborales para el personal sanitario con posible exposición al COVID-19 [Intenet]. USA; 2020. Disponible en: https://www.cdc.gov/coronavirus/2019-ncov/ guidance-risk-assesment-hcp-hml

30. Center for Disease Control and Prevention. Recomendaciones provisionales de prevención y control de infecciones para el personal de atención médica durante la pandemia de la enfermedad por coronavirus 2019 (COVID-19) [Internet]. USA; 2020. Disponible en: https://www.cdc.gov/coronavirus/2019-ncov/hcp/infectioncontrol-recomendations.html
Correspondencia:

Marco Antonio Alfaro Angulo

Dirección: Urb. Juan Pablo II 2.a etapa E1.La Libertad. Trujillo, Perú.

Teléfono: 924730989

Correo electrónico: marcoalfaroa@yahoo.com.mx

Recibido: 09 de junio de 2020.

Evaluado: 10 de noviembre de 2020

Aprobado: 18 de enero de 2021.

( ) La revista. Publicado por Universidad de San Martín de Porres, Perú. (cc) ${ }^{\mathbf{B Y}}$ Licencia de Creative Commons Artículo en acceso abierto bajo términos de Licencia Creative Commons Atribución 4.0 Internacional. (http://creativecommons.org/licenses/by/4.0/)

ORCID iDs

Marco Antonio Alfaro Angulo https: / / orcid.org/0000-0002-6105-4649 